\title{
Study on Plant DNA Methylation through RdDM Pathway and DRM2
}

\author{
Kourosh Mohammadi $^{1}$, Ali Movahedi ${ }^{1}$, Samaneh Sadat Maleki ${ }^{1}$, Qiang Zhuge $^{1 *}$ \\ ${ }^{l}$ Co-Innovation Center for Sustainable Forestry in Southern China, Key Laboratory of Forest Genetics and \\ Biotechnology, Ministry of Education, Nanjing Forestry University, Nanjing 210037, China
}
*Corresponding Author: Qiang Zhuge, The Cooperative Innovation Center of Southern Modern Forestry, Key Laboratory of Forest Genetics and Biotechnology, Ministry of Education, Nanjing Forestry University, Nanjing 210037, China.

\begin{abstract}
DNA information and sequences of plant genomes always need to be stable. Hence, the improvement of a resistant system in plant genomes is necessary to avoid a restriction of conserved DNA sequences. The protection system in stressed plant cells is activated rapidly and dynamically through the RNA directed DNA methylation (RdDM) pathway to control the stress effects before any damage occurs in plant genomes. The RdDM pathway is a key process including short interfering RNA (siRNA), causes epigenetic modification in plant genomes. The modification system of plant cells such as cytosine methylation is associated with histone modification through the different roles in regulating of the gene expression and genome structure. The environmental stresses such as plant pathogenic infections can change the level of DNA methylation in plant genomes. This phenomenon could influence plant ability in more adaptation to different environmental conditions. The DNA methylation in plant cells is facilitated by Domain rearrange DNA methyltransferase (DRM2) which plays a key role in control and manage of the enzymatic process of RdDM pathway. In this study, we provide an overview of function and variation of DNA methylation under different environmental stresses and the key roles of DRM2 in RdDM pathway which complete the pathway reactions through DNA methylation process in plant cells.
\end{abstract}

Keywords: DRM, DNA methylation, RdDM, siRNA

\section{INTRODUCTION}

The control of gene expression and suppression of transposons activity in plant cells are regulated by DNA methylation which is an epigenetic mechanism of gene regulation (zhong et al 2014). The cytosine DNA methylation is containing two phases, initiation and maintenance in plant genomes. The first phase requires a trigger such as a double-stranded RNA. Following DNA replication, the methylation process is supported by copying a pattern of methylation to new strands of DNA and maintains the methylation status. The DNA methylation in plants ideally maintained in order of CG context with cytosine following guanine and in CNG contexts that $\mathrm{N}$ could be any nucleotide except guanine (Wada et al. 2003). Analysis of DNA methylation in some of model plants such as Arabidopsis thaliana shows seven important DNA methyltransferases, containing Domain rearrange DNA methylase 1 (DRM1) and DRM2, chromomethylase 1 (CMT1), CMT2 and CMT3, methyltransferase 1 (MET1) and MET2, and fifteen H3K9 encoding genes (SUVH1-10 and SUVR15) (Law and Jacobsen 2010). Between the mentioned H3K9 methyltransferases, the SU(VAR)3-9 homologous KRYPTONITE (KYP which is known as SUVH4), SUVH5 and SUVH6 are responsible for high methylation level (Bernatavichute et al. 2008). Furthermore, the SUVH2 and SUVH9 couple, which are two catalytically inactive homologues involved in RdDM pathway, are required for heterochromatin formation and are connected to methylation DNA to recruitment of chromatinremodeling complex in target loci (Jing et al. 2016). Additionally, between all of DNA methyltransferases, the DRM2, CMT2, CMT3 and MET1 have been shown to be important in DNA methylation (Du et al. 2015). The comparison of expression level between DNA methyltransferases revealed that DRM2 expression level is much higher than DRMI in tested RNA and the DRM2 seems to be plays a key role and encodes predominant enzyme between DRM gene family members (Ashapkin et al. 2016). Onodera et al. 2005 reported that in plants, de novo DNA methylation is 
involved in post-transcriptional gene silencing (PTGS) or transcriptional gene silencing (TGS) that is activated by biotic or abiotic stress, directed by many 80-nucleotide long non-coding RNAs and 24nucleotide small interfering RNAs (siRNAs). This process is called the RNA-directed DNA methylation (RdDM) pathway. During this enzymatic pathway, the DRM2 is a major de novo DNA methyltransferase enzyme and begins a new methylation pattern (Naumann et al. 2011). All types of DNA methylation in plants such as $\mathrm{CG}, \mathrm{CNN}$ and $\mathrm{CNG}$ are controlled by three methyltransferases MET1 and DRM2 which are two homologues of mammalian DNA methyltransferases (DNMT1 and DNMT3) respectively and CMT3 which is a plant specific chromomethylase 3 (Stroud et al. 2013). Dowen et al. (2012) showed that infection of A. thaliana by biotrophic pathogens such as Pseudomonas syringae PV (Pst) virus is caused mutations in DRMI/2 or CMT3 and the loss of function of these elements in the RdDM pathway. Pathogens can alter the level of DNA methylation and transcription in A. thaliana involving the regulation of coding and non-coding elements (Dowen et al. 2012). The DNA methylation by related enzymes and mechanisms such as (RNAi), can modify and protect plant genomic DNA under environmental stress conditions to avoid excessive genetic recombination and population diversity (Bahadur et al. 2015, Pradhan et al. 2015). Epigenetic mechanism in plant somatic cells also can trigger memorization of the stress experience and transfer the memory to the following generation (Bilichak and Kovalchuk 2016) which is important to improvement and survival of plants.

\section{DNA Methylation in Different STRESS Conditions}

A research on modification of tobacco (Nicotiana tabacum) DNA methylation by different pathogens shows that when NtMET1 methyltansferase in N. tabacum is silenced using an anti-sense RNA technique, the expression of approximately 30 genes is up-regulated by $62.5 \%$. This result is highlighted to the response of plant cells to environmental stresses (Bahadur et al. 2015). Study on the plant cells which artificially were inoculated with tobacco mosaic virus (TMV) to evaluate the levels of transcription, showed that after proximately 24 hours the methylation level of pathogen-responsive genes such as NtAlixl and NtGPDL were altered rapidly by hypersensitivity reactions (HR), following by an alteration of gene expression. Based on this result, change in methylation level causes to change in the expression level of stress response genes, suggest that the viral infections can decrease the level of transcription and methylation in plant genomes (Carbonell and Carrington 2015, Choi and Sano 2007). The effects of low temperatures on plant genomes can also cause to decrease in the value of methyltransferases and the genomic methylation levels. An experiment in temperate fruit crops showed a decrease in the total methylation level under low temperature, from $27.2 \%$ in dormant bud, to $21 \%$ in fruit set stage (Kumar et al. 2016). Another experiment using HPLC in corn (Zea mays L) showed more than $10 \%$ reduction of methylation level in this plant cells (Steward et al. 2002). The cold stress in maize root leads to decrease of methylation level, caused to increase the expression level of ZmMET1 (Zea mays DNA methyltransferase/ MET1) which is containing the part of protein coding regions and retrotransposone-like sequences (steward 2000). Choi and Sano (2007) reported the hypomethylation of $10 \%$ of loci in the genome of tobacco plants under low temperature, which shows that alterations in methylation level can change the level of gene expression. The mapping of methylation shows that the cold stress caused to demethylation in core regions, nevertheless the residues and histone proteins of DNA cytosine methylation have a close cooperation with gene expression and the genomic DNA methylation pattern could potentially be an effective factor in the balance of gene expression (Yang et al. 2015, Choi and Sano 2007, Steward et al. 2002). The higher DNA methylation level of sensitive plants to heavy metals revealed the higher level of methyltransferase activities, in compare to DNA methylation level of resistant plants. For example the Clover (Trifolium repens $L$.) which is sensitive to heavy metals exhibited a higher level of methylation and methyltransferase activities whereas in industrial hemp (Cannabis sativa L.) which is partially resistant to heavy metals the methylation level is not affected (Aina et al. 2004). These results exhibited that high level of methylation can directly affects methyltransferase activities cause to greater resistance to environmental stresses such as heavy metals. Another important mechanism in plant cells is the control of salt concentration and osmotic pressure through water absorption to avoid high concentrations of salt within the plant cells. The water deficiency always causes to drought and salt stresses, because high concentration of salt is difficult to control with less amount of water absorption in plants. For example: the Crassulacean acid metabolism (CAM) plants such as 
MESEMBRYANTHEMUM CRYSTALLINUM, switch to CAM pathway from C3-photosynthesis and close the stomata during the daytime and open at night (Dyachenko et al. 2006). The CAM pathway causes development of CNG hypermethylation of satellite DNA in facultative halophyte Mesembryanthemum crystallinum. Under drought and salt stress conditions switching from C3 to CAM photosynthesis in plant cells causes to reduce the water loss and increase the plant tolerance to high salinity (Naydenov et al. 2015, Dyachenko et al. 2006). DNA methylation is an epigenetic gene regulator system (Zhong et al. 2014) which is important for response to abiotic stresses such as salt and drought in plants (Naydenov et al. 2015). In compare to other epigenetic modification systems such as phosphorylation and acetylation of histones, the cytosine methylation $(5-\mathrm{mC})$ is more vital for protective activities of the plant cells (Vining et al. 2012).

\section{ESSENTIAL ROLE OF DRM2 IN RDDM PATHWAY}

In plants, the de novo DNA methylation completed by DRM2 and RNA interference (RNAi) like machinery, in addition of two plant specific RNA polymerases Pol IV and Pol V, through RNAdirected DNA methylation pathway (Law and Jacobsen, 2010). RdDM pathway is a conserved phenomenon, which is mediated by transcriptional gene silencing in plants, animals and fungi (Zhang et al. 2013a). In plants, RdDM pathway is an essential process for protection against DNA damage to control the transposons and genome stability in stress conditions or nuclease activities and silencing the endogenous genes (Zhang and Zhu 2011). The RdDM pathway is started with a plant-specific RNA polymerase IV (pol IV) in biogenesis phase and directed to chromatin via both of SNF2-like chromatin-remodeling factor (CLSY1) and the SAWADEE HOMEO DOMAIN HOMOLOG 1 (SHH1) that are minor proteins (Smith et al. 2007) (Fig. 1). Then DNA transcription factor 1 (DTF1) associates with SHH1 to detect and methylate histone H3K9, and finds its exact target by polIV (Zhang et al. 2013b; Law et al. 2013). Pol IV transcripts methylated DNA into ssRNA and then convert to dsRNA via the RNA-dependent RNA polymerase 2 (RDR2) and RNA-directed DNA methylation 4 (RDM4) with association of Involved in Denovo 2 (IND2) and RNA-directed DNA methylation 12 (RDM12) proteins (Haag et al. 2012). The dsRNA then cleaves into 24-nt siRNA duplexes and 3' overhangs via Dicer-like endonucleases 3 (DCL3) and recruits Protein HUA ENHANCER 1 (HEN1) which is a methyltransferase enzyme to methylate siRNAs at 3 ' end and prevent their degradation by endonuclease activities (Xie et al. 2004). The product of upstream phase is then transferred to downstream via loading of methylated siRNA duplexes into RNA-induced transcriptional silencing (RITS) to transfers 3 ' overhang duplex siRNA into target mRNA and causes to silencing of expression (Yang et al. 2006). The RITS complex contains AGO4/6/9 proteins and depending on transcription of pol IV from RNA, directs CG and non-CG DNA methylation to target loci on RNA scaffolds by recruiting DDR complex which is an association of proteins, containing Defective in Meristem Silencing 3 (DMS3), DEFECTIVE IN RNA-DIRECTED DNA METHYLATION 1 (DRD1) and RNA-DIRECTED DNA METHYLATION 1 (RDM1) act widely in silencing (Johnson et al. 2014). The interaction between AGO4/6/9 siRNA and pol V is regulated by RDM12/IND2 complex in targeting phase (Ausin et al. 2009). The proteins in DDR complex are not only responsible for silencing defective genes in different area but are associated with each other and used by pol V (Law et al. 2010) to recruits the DRM2 during transcrip-tion of the RNA scaffold and interact with proteins AGO4/6/9 siRNA complex in targeting phase (Zhong et al. 2014). The interaction of DRM2 and other proteins such as AGO4 was confirmed using immunoprecipitation and mass spectrometry (MS) experiments and suggest the DRM2 is tagged with 9xMYC-Biotin Ligase Recognition Peptide (BLRP)-DRM2. After performing MYC pull-down assay DRM2 was isolated using immobilized MYC beads. The presence of AGO4 in purified DRM2 fraction was confirmed by examination of an AGO4 endogenous antibody (Zhong et al. 2014). Study on the nature of the RdDM pathway has shown that completion of this pathway is required to polV association with chromatin. This result suggests that the RdDM is a self-recycling pathway (Johnson et al 2014). The DRM2 manages DNA methylation products to determine the specific target loci through transcription of the RNA scaffold and plays a key role in initiating and balancing of DNA methylation in enzymatic mechanism of RdDM pathway (Zhong et al. 2014; Johnson et al. 2014). The co-occurrence of pol IVdependent siRNA and pol V-dependent noncoding RNA transcripts may cause DRM2 to specify its precise action site (Zhong et al. 2014). Study of plant genomes shows a strong correlation between siRNA and DRM2 during DNA methylation (Law et al., 2013). 


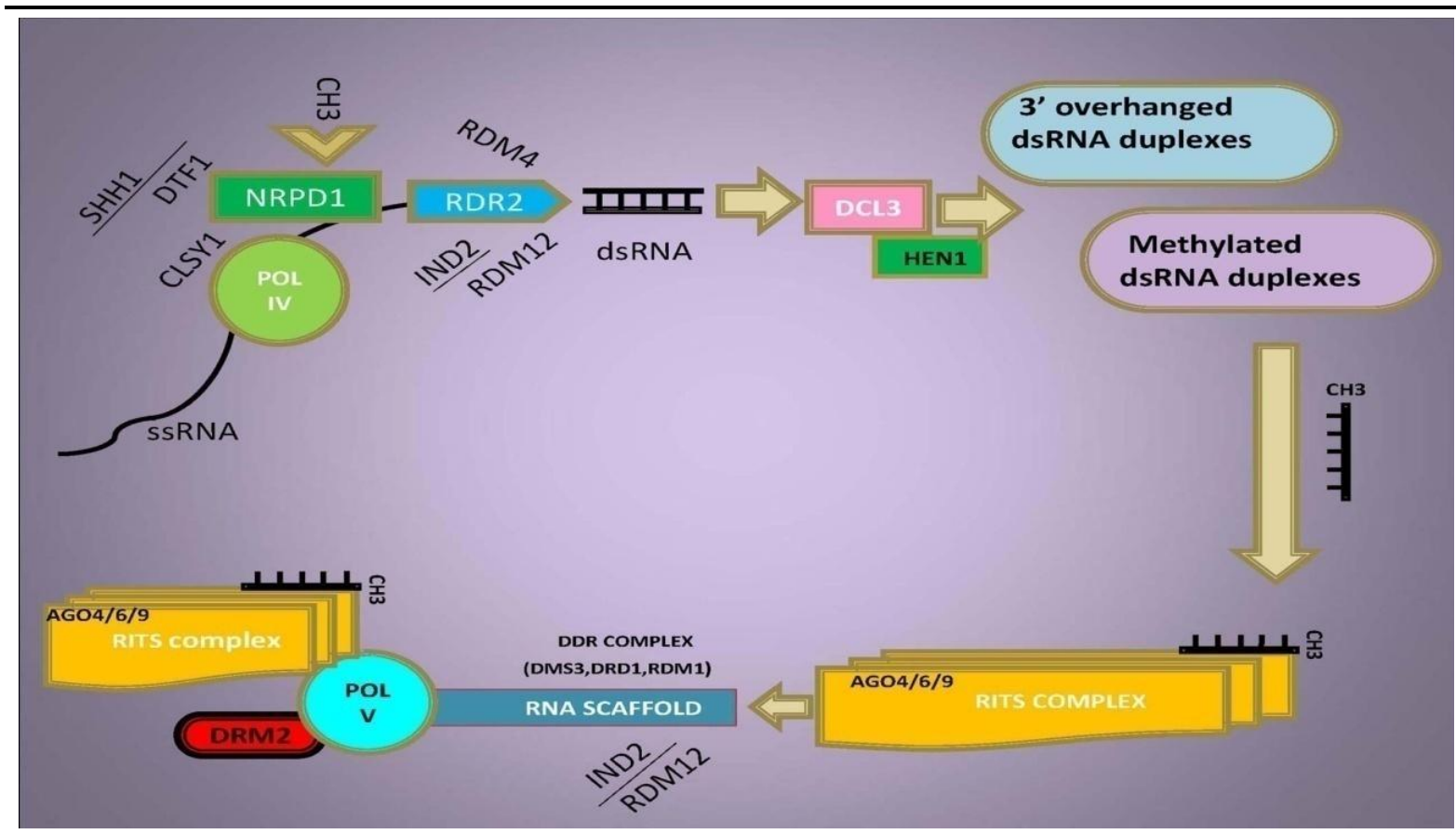

Figure 1: RdDM pathway. The biogenesis phase is initiated by polIV when CLSY1 causes polIV to start RdDM with association of SHH1/DTF1 complex that recruits polIV to chromatin. polIV then transcribes methylated DNA and repetitive sequences into ssRNA which are converted to dsRNA by RDR2 and cooperating activity of IND2/RDM12. DCL3 cleaves dsRNA into 24-nucleotide siRNA duplexes with 3' overhangs and this product methylated by HEN1. Methylated duplexes of biogenesis phase, then converted to one strand, shift to downstream phase and loaded into RITS complex that covers AGO4/6/9. The DDR complex that is containing RDM1, DRD1, and DMS3 also in association with polV and mediation of scaffold RNA, applying DRM2 to interact with AGO proteins to determining the specific target loci and complete the RdDM pathway by DNA methylation.

\section{DISCUSSION}

Principally, we need more understanding in regulation of RdDM pathway and its components. RdDM pathway is an epigenetic process (Matzke et al. 2015), play a vital role in successful plant stress responses and involves microRNAs or 21 nt siRNAs (Xie and $\mathrm{Yu}$ 2015) and important RNA polymerases such as Pol IV (Huang et al. 2017), which initiate the synthesis of siRNAs (Botuyan and Mer 2016), but the most of intercellular reactions of this pathway remain with no clear explain. The $D R M 2$ as a major factor of RdDM pathway manages the DNA methylation to determination of specific target loci through transcription and exhibited a strong correlation with siRNA in methylation process. The DRM2 also plays a key role in initiating and balancing of DNA methylation in all of enzymatic mechanism of RdDM pathway. It is interesting to know the exact mechanism of DRM2 in recognition of the certain loci through transcription of the RNA scaffold in the RdDM pathway. The specific proteins which are responsible to environmental stresses in interaction with DRM2 protein are unknown yet. Moreover, we need to know whether variations of epigenetic marks is controlled by only one specific regulator system or some random events control this process. Plant epigenetic experiments mostly performed using model plants such as Arabidopsis thaliana, but in order to screening of a precise and the novel data in epigenetic variation of plant genomes, the exploration of methylation in different non-model plants is essential.

\section{ACKNOWLEDGMENTS}

This work was supported by the National Science Foundation of China (No. 31570650) and the Priority Academic Program Development of Jiangsu Higher Education Institutions.

\section{REFERENCES}

Aina R. Sgorbati S. Santagostino A. Labra M. Ghiani A. \& Citterio S. 2004. Specific hypomethylation of DNA is induced by heavy metals in white clover and industrial hemp. Physiol Plant. 121: 472-480.

Ashapkin V.V. Kutueva L.I. \& Vanyushin B.F. 2016. Plant DNA methyltransferase genes: Multiplicity, expression, methylation patterns. Biochemistry (Moscow). 81: 141-151. 
Ausin, I. Mockler, T.C. Chory, J. and Jacobsen, S.E. 2009. IDN1 and IDN2 are required for de novo DNA methylation in Arabidopsis thaliana. Nat. Struct. Mol Biol. 16: 1325-1327.

Bahadur B., Rajam M.V., Sahijram L \& Krishnamurthy K.V. 2015. Plant Biology and Biotechnology, Volume II: Plant Genomics and Biotechnology. pp. 265-278. In: Anjana Munshi. Ahuja Y R. \& Bahadur B. Epigenetic mechanism in plants: an overview, Springer India, New Delhi, Heidelberg, New York, Dordrecht, London .

Bernatavichute Y.V. Zhang X. Cokus S. Pellegrini M \& Jacobsen S.E. 2008. Genome-wide association of histone H3 lysine nine methylation with CHG DNA methylation in Arabidopsis thaliana. PloS one. 3: 3156.

Bilichak A. \& Kovalchuk I. 2016. Transgenerational response to stress in plants and its alication for breeding. J Exp Bot. 67: 2081-2092.

Botuyan, M.V. and Mer, G., 2016. Tudor Domains as Methyl-Lysine and Methyl-Arginine Readers. In Chromatin Signaling and Diseases (pp. 149-165).

Carbonell A. \& Carrington J.C. 2015. Antiviral roles of plant ARGONAUTES. Curr opin plant biol 27: 111117.

Choi C.S. \& Sano H. 2007. Abiotic-stress induces demethylation and transcriptional activation of a gene encoding a glycerophosphodiesterase-like protein in tobacco plants. Mol Genet Genom. 277: 589-600.

Dowen R.H. Pelizzola M. Schmitz R.J. Lister R. Dowen J.M. Nery J.R. Dixon J.E. \& Ecker, J.R. 2012. Widespread dynamic DNA methylation in response to biotic stress. P NatL Acad Sci. 109: E2183-E2191.

Du J. Johnson L.M. Jacobsen S.E. \& Patel D.J. 2015. DNA methylation pathways and their crosstalk with histone methylation. Nat Rev Mol Cell Biol. 16: 519-532.

Dyachenko O.V. Zakharchenko N.S. Shevchuk T.V. Bohnert H.J. Cushman J.C. \& Buryanov Y.I. 2006. Effect of hypermethylation of CCWGG sequences in DNA of Mesembryanthemum crystallinum plants on their adaptation to salt stress. Biochemistry (Moscow). 71: 461-465.

Haag J.R. Ream T.S. Marasco M. Nicora C.D. Norbeck A.D. Pasa-Tolic L. \& Pikaard C.S. 2012. In vitro transcription activities of Pol IV, Pol V, and RDR2 reveal coupling of Pol IV and RDR2 for dsRNA synthesis in plant RNA silencing. Mol cell. 48: 811-818.

Huang, J., Lynn, J.S., Schulte, L., Vendramin, S. and McGinnis, K., 2017. Epigenetic control of gene expression in maize. In International review of cell and molecular biology (Vol. 328, pp. 25-48). Academic Press.

Jing Y. Sun H. Yuan W. Wang Y. Li Q. Liu Y. Li Y. and Qian W. 2016. SUVH2 \& SUVH9 couple two essential steps for transcriptional gene silencing in Arabidopsis. Mol plant.

Johnson L.M. Du J. Hale C.J. Bischof S. Feng S. Chodavarapu R.K. Zhong X. Marson G. Pellegrini M. Segal D.J. \& Patel D.J. 2014. SRA-and SET-domain-containing proteins link RNA polymerase V occupancy to DNA methylation. Nature. 507: 124-128.

Kumar G. Rattan U.K. \& Singh A.K. 2016. Chilling-Mediated DNA Methylation Changes during Dormancy and Its Release Reveal the Importance of Epigenetic Regulation during Winter Dormancy in Ale (Malus X domestica Borkh.). PloS one. 11: 149934.

Law J.A. \& Jacobsen S.E. 2010. Establishing, maintaining and modifying DNA methylation patterns in plants and animals. Nat Rev Genet 11: 204-220.

Law J.A. Ausin I. Johnson L.M. Vashisht A.A. Zhu J.K. Wohlschlegel J.A. \& Jacobsen S.E. 2010. A protein complex required for polymerase $\mathrm{V}$ transcripts and RNA-directed DNA methylation in Arabidopsis. Curr Biol. 20: 951-956.

Law J.A. Du J. Hale C.J. Feng S. Krajewski K. Palanca A.M.S. Strahl B.D. Patel D.J. \& Jacobsen S.E. 2013. Polymerase IV occupancy at RNA-directed DNA methylation sites requires SHH1. Nature. 498: 385-389.

Matzke, M.A., Kanno, T. and Matzke, A.J., 2015. RNA-directed DNA methylation: the evolution of a complex epigenetic pathway in flowering plants. Annual review of plant biology, 66, pp.243-267.

Naumann U. Daxinger L. Kanno T. Eun C. Long Q. Lorkovic Z.J. Matzke M. \& Matzke A.J. 2011. Genetic evidence that DNA methyltransferase DRM2 has a direct catalytic role in RNA-directed DNA methylation in Arabidopsis thaliana. Genetics. 187: 977-979.

Naydenov M. Baev V. Apostolova E. Gospodinova N. Sablok G. Gozmanova M. \& Yahubyan G. 2015. Hightemperature effect on genes engaged in DNA methylation and affected by DNA methylation in Arabidopsis. Plant Physiol Biochem. 87: 102-108.

Onodera Y. Haag, J.R. Ream T. Nunes P.C. Pontes, O. \& Pikaard, C.S. 2005. Plant nuclear RNA polymerase IV mediates siRNA and DNA methylation-dependent heterochromatin formation. Cell. 120: 613-622.

Pradhan A. Naik N. \& Sahoo K.K. 2015. RNAi Mediated Drought and Salinity Stress Tolerance in Plants. Am J Plant Sci. 6: 1990. 
Smith L.M. Pontes O. Searle I. Yelina N. Yousafzai F.K. Herr A.J. Pikaard C.S. \& Baulcombe D.C. 2007. An SNF2 protein associated with nuclear RNA silencing and the spread of a silencing signal between cells in Arabidopsis. The Plant Cell. 19: 1507-1521.

Steward N. Ito M. Yamaguchi Y. Koizumi N. \& Sano H. 2002. Periodic DNA methylation in maize nucleosomes and demethylation by environmental stress. J Biol Chem. 277: 37741-37746.

Steward N. Kusano T and Sano H. 2000. Expression of ZmMET1, a gene encoding a DNA methyltransferase from maize, is associated not only with DNA replication in actively proliferating cells, but also with altered DNA methylation status in cold-stressed quiescent cells. Nucleic Acids Res. 28: 3250-3259.

Stroud H. Greenberg M.V. Feng S. Bernatavichute Y.V. \& Jacobsen S.E. 2013. Comprehensive analysis of silencing mutants reveals complex regulation of the Arabidopsis methylome. Cell. 152: 352-364.

Vining K.J. Pomraning K.R. Wilhelm L.J. Priest H.D. Pellegrini, M. Mockler T.C. Freitag M. \& Strauss S.H. 2012. Dynamic DNA cytosine methylation in the Populus trichocarpa genome: tissue-level variation and relationship to gene expression. Bmc Genomics. 13: 1.

Wada Y. Ohya H. Yamaguchi Y. Koizumi N. and Sano H. 2003. Preferential de novo methylation of cytosine residues in non-CpG sequences by a domains rearranged DNA methyltransferase from tobacco plants. J. Biol.Chem. 278: 42386-42393.

Xie Z. Johansen L.K. Gustafson A.M. Kasschau K.D. Lellis A.D. Zilberman D. Jacobsen, S.E. \& Carrington J.C. 2004. Genetic and functional diversification of small RNA pathways in plants. PLoS Biol. 2: 104.

Xie, M. and Yu, B., 2015. siRNA-directed DNA methylation in plants. Current genomics, 16(1), pp.23-31.

Yang H. Chang F. You C. Cui J. Zhu G. Wang L. Zheng Y. Qi J. \& Ma H. 2015. Whole-genome DNA methylation patterns and complex associations with gene structure and expression during flower development in Arabidopsis. Plant J. 81: 268-281.

Yang Z. Ebright Y.W. Yu B. \& Chen X. 2006. HEN1 recognizes 21-24 nt small RNA duplexes and deposits a methyl group onto the 2' $\mathrm{OH}$ of the $3^{\prime}$ terminal nucleotide. Nucleic acids res. 34: 667-675.

Zhang H. \& Zhu J.K. 2011. RNA-directed DNA methylation. Curr Opin plant biol 14: 142-147.

Zhang H. He X. \& Zhu J.K. 2013a. RNA-directed DNA methylation in plants: Where to start?. RNA biol. 10: 1593-1596.

Zhang H. Ma Z.Y. Zeng L. Tanaka K. Zhang C.J. Ma J. Bai G. Wang P. Zhang S.W. Liu Z.W. and Cai T. 2013b. DTF1 is a core component of RNA-directed DNA methylation and may assist in the recruitment of Pol IV. Proceedings of the National Academy of Sciences 110(20): 8290-8295.

Zhong X. Du J. Hale C.J. Gallego-Bartolome J. Feng S. Vashisht, A.A. Chory, J. Wohlschlegel J.A. Patel D.J. \& Jacobsen S.E. 2014. Molecular mechanism of action of plant DRM de novo DNA methyltransferases. Cell. 157: 1050-1060.

Citation: K. Mohammadi et al., "Study on Plant DNA Methylation through RdDM Pathway and DRM2", International Journal of Research studies in Microbiology and Biotechnology, vol. 4, no. 1, p. 11-16, 2018. http://dx.doi.org/10.20431/2454-9428.0401002

Copyright: (C) 2018 Authors. This is an open-access article distributed under the terms of the Creative Commons Attribution License, which permits unrestricted use, distribution, and reproduction in any medium, provided the original author and source are credited. 